\title{
Application of a power system stabiliser and a static VAR controller to a multimachine power system
}

\author{
C.-H. Cheng, MSc \\ Y.-Y. Hsu, PhD
}

Abstract: The damping of the electromechanical mode oscillation in a multimachine power system is improved by a power system stabiliser (PSS). To prevent system voltage profile from being deteriorated by the PSS, a static VAR compensator (SVC) with a self-tuning feedback controller is also developed. The effectiveness of the proposed PSS and SVC is demonstrated by computer simulation of the dynamic responses of the system subject to a three-phase fault. It is observed that the combination of PSS and SVC is capable of increasing system damping without deteriorating system voltage profile.

\section{List of symbols}

$\begin{array}{ll}\omega & =\text { angular speed } \\ \delta & =\text { torque angle } \\ L_{d} & =d \text {-axis stator winding inductance } \\ L_{q} & =q \text {-axis stator winding inductance } \\ L_{F} & =\text { field winding inductance } \\ L_{D} & =d \text {-axis damper winding inductance } \\ L_{Q} & =q \text {-axis damper winding inductance } \\ l_{d} & =\text { leakage inductance of } d \text {-axis stator } \\ & \text { winding } \\ l_{q} & =\text { leakage inductance of } q \text {-axis stator } \\ & \text { winding } \\ k M_{F}=k M_{D} & =M_{R}=L_{d}-l_{d} \\ k M_{Q} & =L_{q}-l_{q} \\ r & =\text { stator resistance } \\ r_{F} & =\text { field resistance } \\ r_{D} & =\text { resistance of } d \text {-axis damper winding } \\ r_{Q} & =\text { resistance of } q \text {-axis damper winding } \\ D & =\text { damping coefficient } \\ T_{A} & =\text { regulator time constant } \\ K_{A} & =\text { regulator gain } \\ T_{F} & =\text { stabilising transformer time constant } \\ K_{F} & =\text { stabilising transformer gain } \\ V_{t} & =\text { generator terminal voltage } \\ V_{R E F} & =\text { reference voltage for generator } \\ T_{m} & =\text { mechanical torque } \\ E_{F D} & =\text { excitor field voltage } \\ V_{S T} & =\text { stabilising transformer output } \\ K_{P S}, K_{I S} & =\text { PSS gains } \\ K_{P}, K_{I}, K_{D} & =\text { PID controller gains for the } S \text { SC } \\ & \end{array}$

Paper 7005C (P9, P11), received 7th March 1989

The authors are with the Department of Electrical Engineering, National Taiwan University, Taipei, Taiwan

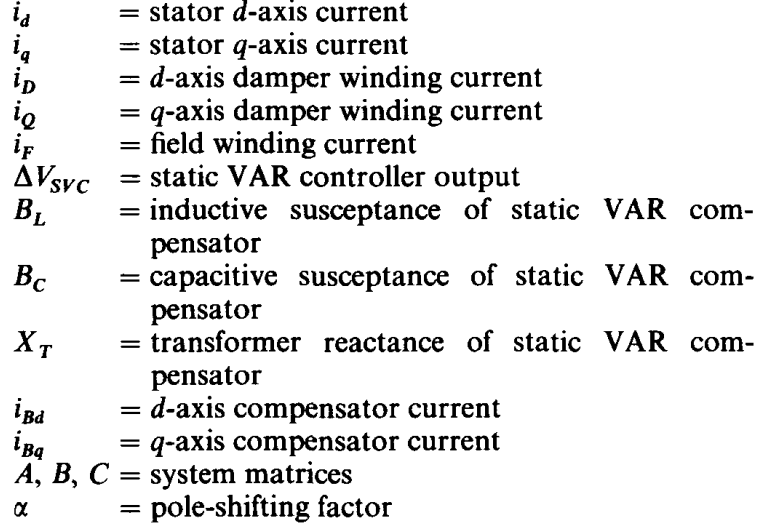

\section{Introduction}

The application of power system stabilisers (PSSs) to improve the damping characteristics of a power system has been extensively investigated over the past two decades [1-5]. Various types of stabiliser such as lead-lag stabilisers [1-3], propositional-integral (PI) stabilisers [6] and variable structure stabilisers [7] have been proposed and found to be effective in damping out system oscillation. Dynamic stability of the power system can thus be improved by these stabilisers. However, it has long been recognised that the PSS, which enhances system damping by incorporating a supplementary signal into the voltage regulator, has a detrimental effect on system voltage profile and may sometimes even cause leading power factor operation of the synchronous generator [8]. Therefore, it is desirable to devise some means to maintain smooth voltage profile while still maintaining the good damping characteristics provided by the PSS.

In the literature, SVC has been successfully used to regulate system voltage [9-11]. Thus, it is decided to choose the SVC as the candidate for maintaining system voltage. The proposed scheme is, then, a combination of a PSS and an SVC which is expected to be capable of both providing system damping and regulating system voltage.

The PSS employed in this paper is a PI stabiliser as suggested in Reference 6. A self-tuning PID feedback controller is developed for the SVC. To demonstrate the effectiveness of the proposed control scheme, digital simulations of the dynamic responses of a multimachine power system to a three-phase fault are carried out. It is found that both system damping and voltage profile can be significantly improved by the proposed controllers. 
Consider the multimachine power system whose one-line diagram is shown in Fig. 1.

The nonlinear synchronous machine equations are

$$
\begin{aligned}
& \delta=\omega-1 \\
& \tau_{j} \dot{\omega}=T_{m}-T_{e}-D \omega \\
& T_{e}=\frac{1}{3}\left(L_{d} i_{q} i_{d}+k M_{F} i_{q} i_{F}+k M_{D} i_{q} i_{D}\right. \\
& \left.-L_{q} i_{d} i_{q}-k M_{Q} i_{d} i_{Q}\right) \\
& V_{d}=-r i_{d}-\omega L_{q} i_{q}-\omega k M_{Q} i_{Q}-L_{d} i_{d} \\
& -k M_{F} i_{F}-k M_{D} i_{D} \\
& -V_{F}=-r_{F} i_{F}-k \dot{M}_{F} \dot{i}_{d}-L_{F} \dot{i}_{F}-M_{R} \dot{i}_{D} \\
& V_{D}=0=-r_{D} i_{D}-k M_{D} \dot{i}_{d}-M_{R} \dot{i}_{F}-L_{D} \dot{i}_{D} \\
& V_{q}=\omega L_{d} i_{d}+\omega k M_{F} i_{F}+\omega k M_{D} i_{D} \\
& -r i_{q}-L_{q} i_{q}-k M_{Q} i_{Q} \\
& V_{Q}=0=-r_{Q} i_{Q}-k M_{Q} \dot{i}_{q}-L_{Q} \dot{i}_{Q}
\end{aligned}
$$

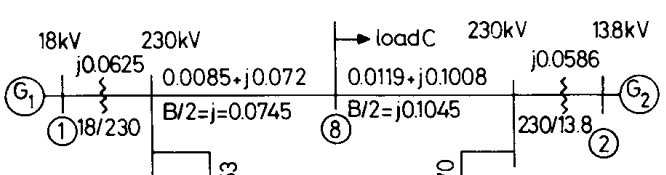

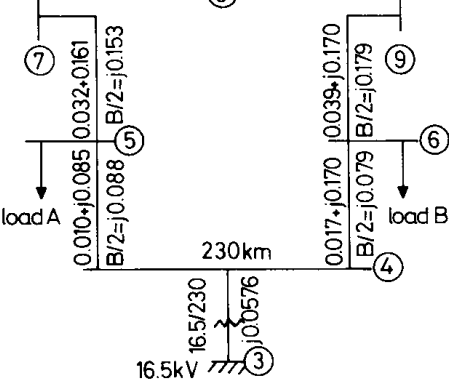

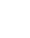

where

$$
\begin{array}{r}
X=\left[\Delta i_{d_{1}}, \Delta i_{F_{1}}, \Delta i_{D_{1}}, \Delta i_{q_{1}}, \Delta i_{Q_{1}}, \Delta i_{d_{2}}, \Delta i_{F_{2}}, \Delta i_{D_{2}},\right. \\
\Delta i_{q 2}, \Delta i_{Q_{2}}, \Delta \omega_{1}, \Delta \delta_{1}, \Delta \omega_{2}, \Delta \delta_{2}, \Delta V_{S T_{1}}, \Delta E_{F D_{1}}, \\
\left.\Delta V_{S T_{2}}, \Delta E_{F D_{2}}, \Delta i_{B d}, \Delta i_{B q}, \Delta B_{L}\right]^{T}
\end{array}
$$

is the state vector. $Y(t)=\Delta \omega(t)$ is the output signal. The control signal $u$ can be expressed in frequency domain as

$$
\begin{aligned}
U(s) & =H_{o}(s) \cdot Y(s) \\
& =\left(K_{P S}+\frac{K_{I S}}{S}\right) \cdot Y(s)
\end{aligned}
$$

where $K_{P S}$ and $K_{I S}$ are the gain of the PI PSS. These unknown parameters $K_{P S}$ and $K_{I S}$ can be determined as follows. Taking the Laplace transform of eqns. 16 and 17, we obtain

$$
\begin{aligned}
S X(s) & =A X(s)+B U(s) \\
Y(s) & =C X(s)
\end{aligned}
$$

Combining eqns. 18, 19 and 20, we have

$$
\left[s I-\left(A+B H_{o}(s) C\right)\right] X(s)=0
$$

The eigenvalues of the closed-loop system equipped with a PI PSS are the solutions of the following characteristic equation

$$
\left|s I-\left(A+B H_{o}(s) C\right)\right|=0
$$

Therefore, the unknown variables $K_{P S}$ and $K_{I S}$ can be obtained by substituting two prespecified eigenvalues $S=\lambda_{1}, \lambda_{2}$ into eqn. (22) and solving the resultant equations. Usually, the prespecified eigenvalues are obtained by shifting two badly-damped eigenvalues leftward. The desired PI PSS parameters from a previous paper [11], $K_{P S}=0.575, K_{I S}=-0.33$ are obtained.

\section{Design of self-tuning PID static VAR controller}

The basic principles underlying the design of the proposed self-tuning PID static VAR compensator can be illustrated by the block diagram in Fig. 2, in which a

$$
\begin{aligned}
& T_{A} \dot{E}_{F D}=-E_{F D}-K_{A} V_{S T}+K_{A}\left(V_{R E F}-V_{t}\right) \\
& T_{F} \dot{V}_{S T}=-V_{S T}+K_{F} \dot{E}_{F D}
\end{aligned}
$$

The equations for the static var compensator are

$$
\begin{aligned}
& V_{d}=L_{B} \dot{i}_{B d}+\omega L_{B} i_{B q} \\
& V_{q}=L_{B} \dot{i}_{B q}-\omega L_{B} i_{B d}
\end{aligned}
$$

where

$$
L_{B}=X_{T}-\frac{1}{B_{C}+B_{L}}
$$

and

$$
B_{L}=B_{L_{o}}+\Delta B_{L}
$$

The control part of SVC can be expressed as

$$
K_{r}\left(\Delta V_{R E F}-\Delta V_{t}+\Delta V_{S V C}\right)=\Delta B_{L}+T_{r} \Delta \dot{B}_{L}
$$

In the design of a fixed-gain PI PSS using the eigenvalueassignment method, the nonlinear differential equations as given above have to be first linearised around the nominal operating condition to obtain the desired state equations

$$
\begin{aligned}
\dot{X}(t) & =A X(t)+B U(t) \\
Y(t) & =C X(t)
\end{aligned}
$$

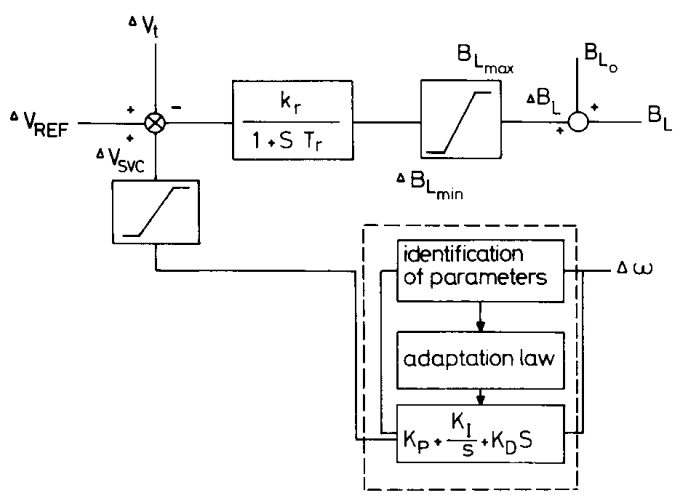

Fig. 2 Block diagram of self-tuning static VAR controller

synchrononous generator with a static VAR compensator (SVC) is equipped with a PID controler whose gain settings $K_{P}, K_{I}$ and $K_{D}$ are adjusted according to the adaptation law described below with the on-line measured generator speed deviation $\Delta \omega$ as the input signal of the controller.

At the sampling instant $t=k T_{s}$, the dynamic behaviour of generator can be described by its input-output 
relationship

$$
A\left(q^{-1}\right) y(k)=B\left(q^{-1}\right) u(k)
$$

where

$$
\begin{array}{ll}
y(k)= & \text { sampled output } \Delta \omega \quad \text { (generator speed } \\
& \text { deviation) at instant } k \\
u(k)= & \text { sampled input } \Delta V_{S V C} \text { of SVC controller } \\
T_{s}= & \text { sampling period } \\
q^{-1}= & \text { backward shift operator } \\
k & =\text { sampling instant } \\
A\left(q^{-1}\right)= & 1+a_{1} q^{-1}+a_{2} q^{-2}+\cdots+a_{n} q^{-n} \\
B\left(q^{-1}\right)= & b_{1} q^{-1}+b_{2} q^{-2}+\cdots+b_{m} q^{-m}
\end{array}
$$

The generator rotor dynamic behaviour can be described by a second-order equation. Therefore, second-order polynomials will be sufficient for $A\left(q^{-1}\right)$ and $B\left(q^{-1}\right)$.

$$
\begin{aligned}
& A\left(q^{-1}\right)=1+a_{1} q^{-1}+a_{2} q^{-2} \\
& B\left(q^{-1}\right)=b_{1} q^{-1}+b_{2} q^{-2}
\end{aligned}
$$

In other words, the input-output relationship can be described by a second-order difference equation

$$
\begin{gathered}
y(k)+a_{1} y(k-1)+a_{2} y(k-2) \\
=b_{1} u(k-1)+b_{2} u(k-2)
\end{gathered}
$$

By continuously measuring the input samples $u(k)$ and output samples $y(k)$, the four coefficients $a_{1}, a_{2}, b_{1}$ and $b_{2}$ can be estimated in real time by using the recursive-leastsquares (RLS) identification method as described in Appendix 7.1.

The gains of the self-tuning PID controller can be obtained using the pole-shifting technique as described in Appendix 7.2.

\section{Results}

The parameters of the system are as follows:

$$
\begin{aligned}
4.1 \text { Synchronous generator } \\
\omega_{R}=377 \mathrm{rad} / \mathrm{s} \\
L_{d}=1.7 \text { p.u. } \\
L_{q}=1.64 \text { p.u. } \\
L_{F}=1.65 \text { p.u. } \\
L_{D}=1.605 \text { p.u. } \\
L_{Q}=1.526 \text { p.u. } \\
k M_{F}=k M_{D}=M_{R}=1.55 \text { p.u. } \\
k M_{Q}=1.49 \text { p.u. } \\
l_{d}=l_{q}=0.15 \text { p.u. } \\
r=0.001096 \text { p.u. } \\
r_{F}=0.000742 \text { p.u. } \\
r_{D}=0.0131 \text { p.u. } \\
r_{Q}=0.054 \text { p.u. } \\
D=0
\end{aligned}
$$

42 Voltage regulator and excitor

$$
\begin{aligned}
T_{A} & =0.05 \mathrm{~s} \\
K_{A} & =400 \\
T_{F} & =1 \mathrm{~s}
\end{aligned}
$$$$
K_{F}=0.025
$$

$$
\begin{aligned}
4.3 \text { Static } & V A R \text { compensator } \\
X_{T} & =0.08 \text { p.u. } \\
K_{r} & =50 \\
T_{r} & =0.15 \mathrm{~s} \\
\Delta V_{S V C_{\max }} & =0.12 \text { p.u. } \\
\Delta V_{S V C_{\min }} & =-0.12 \text { p.u. } \\
\Delta B_{L_{\max }} & =0.06 \text { p.u. } \\
\Delta B_{L_{\min }} & =-0.06 \text { p.u. } \\
L_{B_{o}} & =-0.5 \text { p.u. }
\end{aligned}
$$

$$
\begin{aligned}
& \text { 4.4 Load, KVA } \\
& \text { Load } A=125+\mathrm{j} 40 \\
& \text { Load } B=90+\mathrm{j} 30 \\
& \text { Load } \mathrm{C}=100+\mathrm{j} 30
\end{aligned}
$$

To demonstrate the effectiveness of the proposed controller, a digital simulator was built on a VAX-11/780 minicomputer using the nonlinear system model including the synchronous machines, the excitation system, the static VAR compensators, PSS, and the power system as described in Section 2. Runge-Kutta method was employed to solve the resulting nonlinear differential equations and to simulate the system reponses to the disturbance.

The dynamic responses of the system subject to a fourcycle, three-phase fault taking place at busbar 7 are shown in Figs. 3-6. It is noted that the PSS, which uses the speed deviation of generator 1 as input signal, generates a supplementary damping signal which is fed into the excitation system of generator 1 . The SVC is installed at the armature terminal of generator 1 .

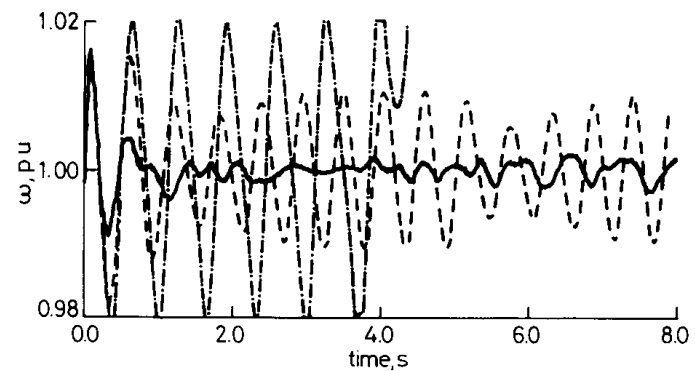

Fig. 3 Speed deviation at generator 1

- - - without SVC and PSS

$\ldots$ with SVC only

with both SVC and PSS

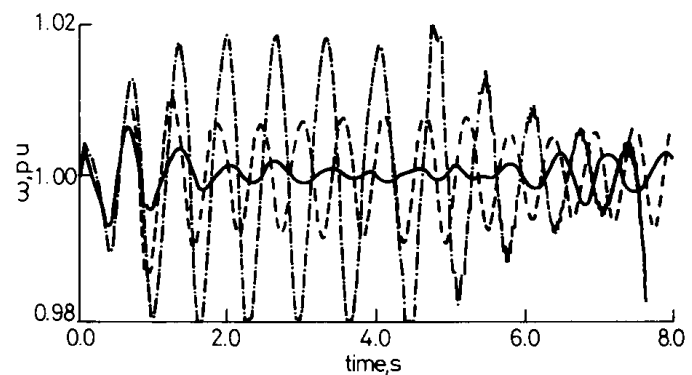

Fig. 4 Speed deviation at generator 2

--- without SVC and PSS

-.- with SVC only

- with both SVC and PSS

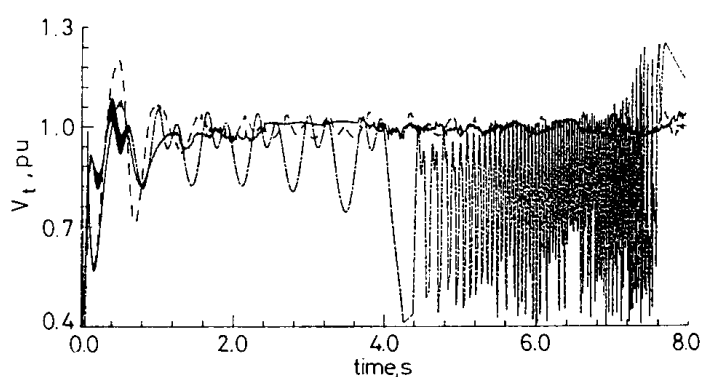

Fig. 5 Terminal voltage deviation at generator 1 without SVC and PSS with SVC only with both SVC and PSS

IEE PROCEEDINGS, Vol. 137, Pt. C, No. 1, JANUARY 1990 
From the response curves in Figs. 3-6, the following observations are in order:

(i) As evidenced by the response curves in Figs. 3-6, the original system without SVC and PSS is unstable.

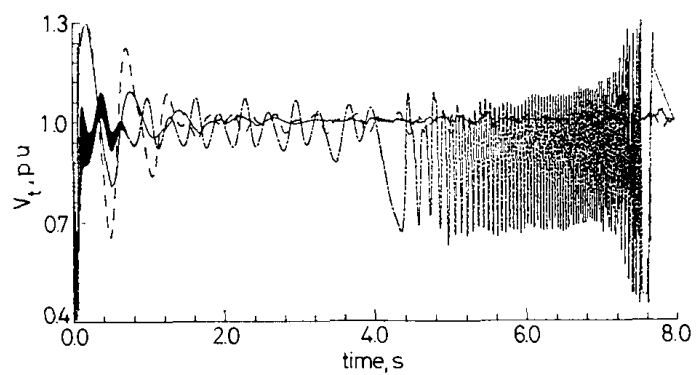

Fig. 6 Terminal voltage deviation at generator 2

-.... without SVC and PSS with SVC only with both SVC and PSS

(ii) The system becomes stable when the SVC is incorporated into the system. As shown in Figs. 3-6, very smooth terminal voltages can be achieved for both generator 1 and generator 2 . However, poor generator speed deviations are still observed with the application of the self-tuning SVC to the system.

(iii) The undesirable large, poorly-damped generator speed oscillations can be effectively damped by further application of the PSS to the system.

(iv) It is noted that a second-order representation of the mechanical system was employed since only the problem of low frequency electromechanical mode oscillation was addressed. To prevent the system from exciting high frequency torsional oscillations, a low pass filter in front of the PSS is necessary [15]. Furthermore, to study the effect of high frequency modes on system dynamic performance, a much more detailed model is required for the shaft system [16-17].

\section{Conclusions}

Design of a power system stabiliser (PSS) and a static VAR compensator (SVC) with a self-tuning PID controller has been presented in this paper. The PSS is capable of providing system damping and the SVC can be used to regulate system voltage. With the combination of the PSS and SVC, the electromechanical mode oscillations can be effectively damped without deteriorating system voltage profile. It is found that system stability can be significantly improved by the proposed control scheme.

\section{References}

1 DEMELLO, F.P., NOLAN, P.J., LASKOWSKI, T.F., and UNDRILL, J.M.: 'Coordinated application of stabilisers in multimachine power systems', IEEE Trans., 1980, PAS-99, pp. 892-901

2 LARSEN, E.V., and SWANN, D.A.: 'Applying power system stabilisers', IEEE Trans., 1981, PAS-100, pp. 3017-3046

3 DEMELLO, F.P., and CONCORDIA, C.: 'Concepts of synchronous machine stability as affected by excitation control', IEEE Trans., 1969, PAS-88, pp. 316-329

4 HSU, Y.Y., SHYUE, S.W., and SU, C.C.: 'Low frequency oscillations in longitudinal power systems: experience with dynamic stability of Taiwan power system', IEEE Trans., 1987, PWRS-2, pp. 92-100

5 HSU, Y.Y., and SU, C.C.: 'Application of power system stabiliser on a power system with pumped storage plant', IEEE Trans., 1988, PWRS-3, pp. 80-86
6 HSU, Y.Y., and HSU, C.Y.: 'Design of a proportional-integral power system stabiliser' IEEE Trans., 1986, PWRS-1, (2) pp. 46-53

7 CHAN, W.C., and HSU, Y.Y.: 'An optimal variable structure stabiliser for power system stabilisation', IEEE Trans., 1983, PAS-102, pp. 1738-1746

8 LIN, C.J., CHEN, Y.T., and CHOU, Y.Y.: 'A study of power system stabiliser selection for Taipower system'. Proc. 5th ROC Symposium on Electrical Power Engineering, 1984, pp. 403-437

9 HAUTH, R.L., HUMANN, T., and NEWELL, R.J.: 'Application of a static VAR system to regulate system voltage in western Nebraska', IEEE Trans., 1978, PAS-97, pp. 1955-1964

10 BRUCOLI, M., TORELLI, F., and TROVATO, M.: 'A decentralised control strategy for dynamic shunt VAR compensation in interconnected power systems', IEE Proc. C, 1985, 132, pp. 229-236

11 HSU, Y.Y., LIU, C.S., LIN, C.J., and HUANG, C.T.: 'Application of power system stabilisers and static VAR compensators on a longitudinal power system'. Paper 88 WM 170-3, presented at the IEEE/PES 1988 Winter Meeting

12 HSU, Y.Y., and CHENG, C.H.: 'Variable structure and adaptive control of a synchronous machine', IEEE Trans., 1988, AES-24, pp. 337-345

13 ASTROM, K.J., and WITTENMARK, B.: 'Self-tuning controllers based on pole-zero placement', IEE Proc. D, 1980, 126, pp. $120-130$

14 WITTENMARK, B., and ASTROM, K.J.: 'Simple self-tuning controllers', in 'Methods and Applications in adaptive control' (Springer-Verlag, New York, 1980), pp. 21-30

15 HSU, Y.Y., and WU, C.J.: 'Decoupled design of static VAR compensators and power system stabilisers for the damping of subsynchronous oscillations using digital filter'. Paper 89 WM 153-8 PWRS, presented at the IEEE/PES PES 1989 Winter Meeting, New York

16 BALDA, J.C., HARLEY, R.G., and EITELBERG, E.: 'Damping of torsional shaft oscillations in two neighbouring turbogenerators', IEEE Trans., 1987, EC-2, pp. 108-115

17 WANG, L., and HSU, Y.Y.: 'Damping of subsynchronous resonance using excitation controllers and static VAR compensators: a comparative study', IEEE Trans., 1988, EC-3, pp. 6-13

\section{$7 \quad$ Appendix}

\subsection{Recursive-least-squares (RLS) identification} method

Rewrite eqn. 26 in vector form

$$
y(k)=\phi^{T}(k) \hat{\theta}(k-1)
$$

where

$$
\phi(k)=[y(k-1), y(k-2), u(k-1), u(k-2)]^{T}
$$

is the data vector and

$$
\hat{\theta}(k-1)=\left[\begin{array}{llll}
a_{1} & a_{2} & b_{1} & b_{2}
\end{array}\right]^{T}
$$

is the parameter vector. The recursive formula is given by

$$
\hat{\theta}(k)=\hat{\theta}(k-1)+H(k-1)\left[y(k)-\phi^{T}(k) \hat{\theta}(k-1)\right]
$$

where the correction vector $H(k-1)$ is given by

$$
H(k-1)=\frac{1}{\phi^{T}(k) K(k-1) \phi(k)+\lambda} K(k-1) \phi(k)
$$

and

$$
K(k-1)=\left[I-H(k-2) \phi^{T}(k-1)\right] K(k-2) / \lambda
$$

The constant $\lambda$ is a forgetting factor very close to unity. A value of 0.98 is found to be satisfactory for the present case.

To start the recursive formula, we set

$$
K(0)=\rho I
$$

where $\rho$ is a great scaling factor.

\subsection{Pole shifting technique for the design of self-tuning PID controller}

In the design of a self-tuning PID controller using the pole-shifting technique, the poles of the open-loop system are first obtained by solving the open-loop characteristic 
equation from eqn. 24

$$
A\left(q^{-1}\right)=1+a_{1} q^{-1}+a_{2} q^{-2}
$$

These open-loop poles are then shifted toward the origin of the unit circle by a pole-shifting factor $\alpha$, through the application of a control signal $u(k)$ of the form

$$
u(k)=\frac{T\left(q^{-1}\right)}{R\left(q^{-1}\right)} y_{r}(k)-\frac{S\left(q^{-1}\right)}{R\left(q^{-1}\right)} y(k)
$$

where $y_{r}(k)$ is reference signal for $y(k)$. This implies that the resulting closed-loop poles will be the roots of the characteristic equation

$$
\begin{aligned}
p\left(q^{-1}\right) & =A\left(\alpha q^{-1}\right)=0 \quad 0 \leqslant \alpha \leqslant 1 \\
& =1+a_{1} \alpha q^{-1}+a_{2} \alpha^{2} q^{-2}
\end{aligned}
$$

Combining eqns. 23 and 34 , we have the transfer function for the closed-loop system

$$
\frac{y(k)}{y_{r}(k)}=\frac{T\left(q^{-1}\right) B\left(q^{-1}\right)}{A\left(q^{-1}\right) R\left(q^{-1}\right)+B\left(q^{-1}\right) S\left(q^{-1}\right)}
$$

Under the assumptions made in Reference $12, B$ can be factorised as

$$
B\left(q^{-1}\right)=B_{1}\left(q^{-1}\right) B_{2}\left(q^{-1}\right)
$$

where all the zeros of $B_{1}$ are outside the restricted stability region and $B_{2}$ are inside the stability region. Comparing the denominators of eqn. 36 after pole-zero cancellation with eqn. 35 , we obtain [11]

$$
A\left(q^{-1}\right) R_{1}\left(q^{-1}\right)+B_{1}\left(q^{-1}\right) S\left(q^{-1}\right)=P\left(q^{-1}\right)
$$

where the two terms $R_{1}\left(q^{-1}\right)$ and $B_{1}\left(q^{-1}\right)$ are derived from pole-zero cancellation.

Substituting eqn. 37 into eqn. 38 , we get

$$
A\left(q^{-1}\right) R\left(q^{-1}\right)+B\left(q^{-1}\right) S\left(q^{-1}\right)=P\left(q^{-1}\right) B_{2}\left(q^{-1}\right)
$$

In order to obtain a self-tuning controller of PID structure, let us assume

$$
\begin{aligned}
R\left(q^{-1}\right) & =\left(1+r_{1} q^{-1}\right)\left(1-q^{-1}\right) \\
S\left(q^{-1}\right) & =S_{0}+S_{1} q^{-1}+S_{2} q^{-2} \\
B_{2}\left(q^{-1}\right) & =1+\alpha q^{-1} \\
T\left(q^{-1}\right) & =S_{0}+S_{1}+S_{2}
\end{aligned}
$$

when eqns. $24,25,40,41$, and 42 are substituted into eqn. 39 , we get

$$
\begin{aligned}
& \left(1+a_{1} q^{-1}+a_{2} q^{-2}\right)\left(1+r_{1} q^{-1}\right)\left(1-q^{-1}\right) \\
& +\left(b_{1} q^{-1}+b_{2} q^{-2}\right)\left(S_{0}+S_{1} q^{-1}+S_{2} q^{-2}\right) \\
& =\left(1+a_{1} \alpha q^{-1}+a_{2} \alpha^{2} q^{-2}\right)\left(1+\alpha q^{-1}\right)
\end{aligned}
$$

Comparing the coefficients on both sides of eqn. 44 , we have

$$
\begin{aligned}
& r_{1}+b_{1} S_{0}=1-a_{1}+\alpha+a_{1} \alpha \\
& r_{1}\left(a_{1}-1\right)+b_{1} S_{1}+b_{2} S_{0}=a_{1}-a_{2}+a_{1} \alpha^{2} \\
& r_{1}\left(a_{2}-a_{1}\right)+b_{2} S_{1}+b_{1} S_{2}=a_{2}+a_{2} \alpha^{3} \\
& -a_{2} r_{1}+b_{2} S_{2}=0
\end{aligned}
$$$$
r_{1}\left(a_{1}-1\right)+b_{1} S_{1}+b_{2} S_{0}=a_{1}-a_{2}+a_{1} \alpha^{2}+a_{2} \alpha^{2}
$$

With $a_{1} a_{2}, b_{1}$, and $b_{2}$ estimated using the RLS identifier, the four parameters $r_{1}, S_{0}, S_{1}$, and $S_{2}$ may be obtained by solving eqns. $45-48$. It is worth noting that, under disturbance conditions, these parameters will change with variations in the estimated values of $a_{1}, a_{2}$, $b_{1}$ and $b_{2}$.

Substituting eqns. $40-43$ into eqn. 34 , we obtain the input signal for the self-tuning controller

$$
\begin{aligned}
u(k)= & \frac{S_{0}+S_{1}+S_{2}}{\left(1+r_{1} q^{-1}\right)\left(1-q^{-1}\right)} y_{r}(k) \\
& -\frac{S_{0}+S_{1} q^{-1}+S_{2} q^{-2}}{\left(1+r_{1} q^{-1}\right)\left(1-q^{-1}\right)} y(k)
\end{aligned}
$$

Recall that a PID controller can be described by [13]

$$
\begin{aligned}
u(k)= & \frac{-T_{s} K_{1}}{\left(1-q^{-1}\right)\left(1+r_{1} q^{-1}\right)}\left(y_{r}(k)-Y(k)\right)+K_{p} y(k) \\
& +\frac{K_{D}\left(1-q^{-1}\right)}{T_{s}\left(1+r_{1} q^{-1}\right)} y(k)
\end{aligned}
$$

In this paper, the reference signal of $y, y_{r}$, is zero because $\Delta \omega$ is used for the output signal $y$. Comparing eqn. 49 with eqn. 50 we have the gains of the self-tuning PID controller

$$
\begin{aligned}
K_{P} & =\left(S_{1}+2 S_{2}\right) /\left(1+r_{1}\right) \\
K_{I} & =-\left(S_{0}+S_{1}+S_{2}\right) / T_{s} \\
K_{D} & =\left\{\left[r_{1} S_{1}-\left(1-r_{1}\right) S_{2}\right] /\left(1+r_{1}\right)\right\} T_{s}
\end{aligned}
$$

These gain settings are computed at each sampling instant using the present estimated values of the four coefficients, $a_{1}, a_{2}, b_{1}$, and $b_{2}$, characterising generator dynamic behaviour at that instant. 